\title{
MODULES WHOSE PROJECTION INVARIANT SUBMODULES HAVE PROJECTION INVARIANT CLOSURES
}

\author{
YELIZ KARA
}

Received 14 March, 2017

\begin{abstract}
We elaborate the class of $P C$-modules in which every projection invariant submodules has projection invariant closures in this study. We provide examples that the class of $P C$ modules does not belong to the system of generalization of extending modules. Moreover, we clarify direct sums and direct summands properties for the former class of modules. It is proved that the aforementioned property is not closed under direct sums. Thereupon, we cope with when the direct sums of $P C$-modules enjoy with the property.
\end{abstract}

2010 Mathematics Subject Classification: 16D10; 16D50

Keywords: complement submodule, extending module, projection invariant submodule

\section{INTRODUCTION}

Let $R$ be a ring with unity and $M$ a unitary right $R$-module. A submodule $K$ of $M$ is called a complement in $M$ if $K$ has no proper essential extension in $M$. Recall that a module $M$ is called extending (or $C S$ ) if every submodule $N$ of $M$ is essential in a direct summand $M$; or every complement submodule of $M$ is a direct summand of $M[4,15]$. A submodule $N$ of $M$ is called a projection (fully) invariant if $f(N) \subseteq N$ for all $f^{2}=f \in \operatorname{End}\left(M_{R}\right)\left(f \in \operatorname{End}\left(M_{R}\right)\right)$. It is well known that every fully invariant submodule is projection invariant (see, [5, page 50]). There are many examples of projection invariant submodules in different algebraic structures. Motivating on this class of submodules, a module $M$ is said to be $\pi$-extending [3], if every projection invariant submodule of $M$ is essential in a direct summand of $M$. It is shown that the class of $\pi$-extending modules is closed under direct sums, but not direct summands (see, [3, Example 5.5], [14, Example 4]). Hence it is investigated some special subclasses of $\pi$-extending modules. To this end, a module $M$ is called strongly $\pi$-extending [9], if every projection invariant submodule of $M$ is essential in a fully invariant direct summand of $M$. It is proved that the class of strongly $\pi$-extending modules is a proper subclass of $\pi$-extending modules.

Let $N$ be a submodule of $M$. A submodule $T$ of $M$ is called a closure (or essential closure) of $N$ in $M$, if $N \leq_{e} T \leq_{c} M$. It is renowned that, every submodule has a closure (see, [15, Proposition 2.5]). Consequently, we concern with closure 
properties of projection invariant submodules. Thereby, we call a module $M$ is projection invariant closure module (denoted, $P C$-module), if every projection invariant submodule of $M$ has a projection invariant closure in $M$. We observe indecomposable modules, uniform modules and nonsingular modules are the examples of $P C$ modules. Moreover it is shown that the $P C$ condition is more general than strongly $\pi$-extending property. Although strongly $\pi$-extending property implies $\pi$-extending condition, we provide by counter examples that the classes of $P C$-modules and $\pi$ extending modules are incomparable. Apart from that, we delve into the direct sums and direct summands properties of $P C$-modules. We prove that projection invariant direct summands of $P C$-modules are $P C$-modules. Even so the aforementioned property is not closed under direct sums. Therefore we show when the direct sums of $P C$-modules is $P C$-module. To this end, a module $M$ has complement sum property, $C S P$ [7], if the sum of every pair of complements of $M$ is a complement of $M$. Furthermore we get the hang of CSP condition which is not Morita invariant. Additionally, we characterize that the extending and quasi-continuous conditions are equivalent for a module with CSP.

For notation, we use $M_{n}(R)$ and $R^{n}$ for the full $n$-by- $n$ matrix ring over $R$ and the direct sum of $n$ copies of $R$ for any positive integer $n$, respectively. For a nonempty subset $X$ of $M, X \leq M, X \leq_{e} M, X \leq_{c} M, X \leq_{d} M$ and $X \unlhd_{p} M$ denote the submodule of $M$, the essential submodule of $M$, the complement submodule of $M$, the direct summand of $M$ and the projection invariant submodule of $M$, respectively. For unknown terminology and notation, see $[1,4,10,11]$.

\section{Preliminary Results}

We locate $P C$-modules with the other renowned classes of modules (e.g., $\pi$ extending, strongly $\pi$-extending) in this section. Observe that indecomposable modules and uniform modules are the examples of $P C$-modules. Furthermore, nonsingular modules have the foregoing property as shown in the first result.

Lemma 1. Every nonsingular module is a PC-module.

Proof. Let $M_{R}$ be a nonsingular module and $X \unlhd_{p} M$. Then $X \leq_{e} T \leq_{c} M$ for some submodule $T$ of $M$. Since $M_{R}$ is nonsingular and $X \unlhd_{p} M, T$ is a projection invariant submodule of $M$ by [3, Lemma 2.3]. Hence $M$ is a $P C$-module.

Let $M_{\mathbb{Z}}=\mathbb{Z} / \mathbb{Z} p$ for any prime $p$. It is clear that $M_{\mathbb{Z}}$ is not nonsingular, but it is a $P C$-module. It follows that the converse of above lemma is not true. Moreover, there are examples which show that the $P C$ condition does not imply uniform or indecomposable properties. For example, let $M_{\mathbb{Z}}=(\mathbb{Z} \oplus \mathbb{Z})_{\mathbb{Z}}$. It is renowned that $M_{\mathbb{Z}}$ is nonsingular, and hence it is a $P C$-module by Lemma 1 . However $M_{\mathbb{Z}}$ is neither uniform nor indecomposable. The next result identifies the connection between the classes of strongly $\pi$-extending modules and $P C$-modules. 
Lemma 2. If $M_{R}$ is a strongly $\pi$-extending module, then $M_{R}$ has $P C$ condition. But the converse need not to be true.

Proof. Let $M_{R}$ be a strongly $\pi$-extending module and $N \unlhd_{p} M$. Then $N \leq_{e}$ $D \leq_{d} M$ for some fully invariant direct summand $D$ of $M$. It follows that $N \leq_{e}$ $D \leq_{c} M$ where $D \unlhd_{p} M$. Therefore $M_{R}$ is a $P C$-module. On the other hand, for the converse, let $R$ be a domain which is not right Ore. Since $R_{R}$ is a prime ring, every nonzero ideal of $R_{R}$ is essential in $R_{R}$. Thus $R_{R}$ is a $P C$-module, because $R$ is an indecomposable $R$-module. However $R_{R}$ is not uniform, so $R$ is not $\pi$-extending and hence it is not strongly $\pi$-extending by [9, Corollary 2.4].

It is interpreted that every strongly $\pi$-extending module is $\pi$-extending in [9]. In connection with the above lemma one might ask that are there any implications between $P C$-modules and $\pi$-extending modules? Now, we get across the following counter examples for the former question.

Example 1. ( $i$ ) Consider $M_{\mathbb{Z}}=\prod_{i \in I} A_{i}$ where $A_{i}=\mathbb{Z}$ for $i \in I$. Note that the Specker group $M_{\mathbb{Z}}$ is not $\pi$-extending by [5]. On the other hand, $M_{\mathbb{Z}}$ is a nonsingular by [6, Proposition 1.22]. Thereupon $M_{\mathbb{Z}}$ is a PC-module by Lemma 1.

(ii) Let $M_{\mathbb{Z}}=\mathbb{Z} \oplus(\mathbb{Z} / \mathbb{Z} p)$ for any prime $p$. Then $M_{\mathbb{Z}}$ is $\pi$-extending which is not strongly $\pi$-extending by [9, Corollary $2.4(i v) \nRightarrow(i)]$. Hence $M_{\mathbb{Z}}$ is not a PC-module by Proposition 1(i).

Note that a ring $R$ is called Abelian if every idempotent of $R$ is central. The next fact provides the implications between the classes of $\pi$-extending modules, strongly $\pi$-extending modules and $P C$-modules under some additional conditions.

Proposition 1. (i) Let $M_{R}$ be a PC-module. Then $M_{R}$ is a $\pi$-extending module if and only if $M_{R}$ is a strongly $\pi$-extending module.

(ii) Let $S=\operatorname{End}\left(M_{R}\right)$ be an Abelian ring. If $M_{R}$ is a $\pi$-extending, then $M_{R}$ is a PC-module.

(i i i ) If $M_{R}$ be a PC-module and every projection invariant essentially closed submodule of $M$ is a fully invariant direct summand, then $M_{R}$ is strongly $\pi$-extending.

Proof. ( $i$ ) Let $M_{R}$ be a $\pi$-extending module and $X \unlhd_{p} M$. Then $X \leq_{e} K \leq_{d} M$ for some direct summand $K$ of $M$. Since $M_{R}$ is a $P C$-module, $K$ is projection invariant in $M$. Hence $K$ is a fully invariant submodule of $M$ by [5, page 50]. Thus $M$ is a strongly $\pi$-extending module. The converse follows by [9, Corollary 2.4].

(ii) Let $X \unlhd_{p} M$. Then $X \leq_{e} K \leq_{d} M$ for some submodule $K$ of $M$. Since $S$ is Abelian, it can be easily seen that every direct summand of $M$ is projection invariant in $M$. Therefore $M$ is a $P C$-module.

(iii) Let $M$ be a $P C$-module and $X \unlhd_{p} M$. Then there exists a projection invariant submodule $K$ of $M$ such that $X \leq_{e} K \leq_{c} M$. By hypothesis, $K$ is a fully invariant direct summand, so $M$ is strongly $\pi$-extending. 
Notice that $M_{\mathbb{Z}}=(\mathbb{Z} / \mathbb{Z} p) \oplus \mathbb{Q}$ is a $P C$-module for any prime $p$. Indeed, it is clear from [8, Example 2.14], $M_{\mathbb{Z}}$ is a $\pi$-extending module with an Abelian endomorphism ring. Thereby, $M_{\mathbb{Z}}$ is a $P C$-module by Proposition 1(i $i$ ). This example and Example 1( $i \mathrm{i}$ ) show that any submodules of a $P C$-module need not to be a $P C$-module, in general.

One might wonder whether $P C$-condition belongs to the system of generalization of extending modules or not. Hence we supply examples which simplify that there is no implication between the classes of $P C$-modules and extending modules.

Example 2. ( $i$ ) Let $R=\left[\begin{array}{ll}\mathbb{Z} & \mathbb{Z} \\ 0 & \mathbb{Z}\end{array}\right]$ be the upper triangular 2-by-2 matrix ring over $\mathbb{Z}$. It is follows that $R_{R}$ is not extending by [15, Example 3.84]. Since $R_{R}$ is nonsingular, it is a $P C$-module by Lemma 1.

(ii) Let $S_{3}$ be the symmetric group on the letter $\{1,2,3\}$ and $\mathbb{Z}_{3}$ the ring of integers modulo 3. Let $R=\mathbb{Z}_{3}\left[S_{3}\right]$ be the group ring of the group $S_{3}$ over $\mathbb{Z}_{3}$. Then $R$ is right self-injective. Thus $R_{R}$ is extending, and hence it is $\pi$-extending. However $R_{R}$ is not a strongly $\pi$-extending module by [2, Example 1.1] and [9, Corollary 2.4]. Now assume that $R_{R}$ is a $P C$-module. Then $R_{R}$ is not $\pi$-extending by Proposition 1(i), a contradiction. Therefore $R_{R}$ is not a $P C$-module.

Recall from [12], a module $M$ is called $U C$-module, if every submodule of $M$ has a unique closure. Following the idea in [12], it is natural to think of unique projection invariant closure module (denoted, $U P C$-module) in which every projection invariant submodule has a unique projection invariant closure.

Observe that every nonsingular and $P C$-module are the examples of $U P C$-modules. However basically the results and their proofs arise out of just simple motifications of the result in [12]. To this end, Example 1( $i$ i $)$ and Example 2(ii) identify that projection invariant submodules need not to have a unique projection invariant closure.

\section{DIRECT SUMS AND DIRECT SUMMANDS}

In this section, our main goal is to deal with the direct summand and direct sum properties of $P C$-module.

Proposition 2. Let $M$ be a $P C$-module such that $M=M_{1} \oplus M_{2}$ where $M_{1}$ and $M_{2}$ are projection invariant submodules of $M$. Then $M_{1}$ and $M_{2}$ are $P C$-modules.

Proof. Let $M=M_{1} \oplus M_{2}$ for some projection invariant $M_{1}, M_{2} \leq M$ and let $X_{1}$ be a projection invariant submodule of $M_{1}$. Then $X_{1} \oplus M_{2}$ is a projection invariant submodule of $M$ by [3, Lemma 4.13]. Hence $X_{1} \oplus M_{2} \leq_{e} K \leq_{c} M$ for some projection invariant submodule $K$ of $M$. Since $K \unlhd_{p} M, K=\left(K \cap M_{1}\right) \oplus\left(K \cap M_{2}\right)$ where $K_{1}=K \cap M_{1} \unlhd_{p} M_{1}$ and $K_{2}=K \cap M_{2} \unlhd_{p} M_{2}$ by [5, page 50]. Hence we obtain $X_{1}=M_{1} \cap\left(X_{1} \oplus M_{2}\right) \leq_{e} K \cap M_{1}=K_{1}$. It is easy to see that $K_{1} \leq_{c} M$. It follows from [10, Proposition 6.24 (1)] that $K_{1} \leq_{c} M_{1}$. Therefore $M_{1}$ is a $P C$ module. Similarly, it can be seen that $M_{2}$ is a $P C$-module. 
Example 1(i i ) examines that the direct sums of $P C$-modules need not to be $P C$ module. To this end, we determine when the class of $P C$-modules is closed under direct sums.

Proposition 3. Let $M=M_{1} \oplus M_{2}$ be an extending module for some $M_{1}, M_{2} \leq$ $M$. If $M_{1}$ and $M_{2}$ are PC-modules, then $M$ is a PC-module.

Proof. Let $Y \unlhd_{p} M$. Then $Y=\left(Y \cap M_{1}\right) \oplus\left(Y \cap M_{2}\right)$ where $Y_{1}=Y \cap M_{1} \unlhd_{p} M_{1}$ and $Y_{2}=Y \cap M_{2} \unlhd_{p} M_{2}$ by [5, page 50]. Hence there exist projection invariant submodules $K_{1}$ of $M_{1}$ and $K_{2}$ of $M_{2}$ such that $X_{1} \leq_{e} K_{1} \leq_{c} M_{1}$ and $X_{2} \leq_{e} K_{2} \leq_{c}$ $M_{2}$. It follows that $M_{1}$ and $M_{2}$ are extending by [11, Proposition 2.7]. Thus $K_{1}$ and $K_{2}$ are direct summands of $M_{1}$ and $M_{2}$, respectively. Consequently $K_{1} \oplus K_{2}$ is a direct summand of $M$, so $K_{1} \oplus K_{2}$ is a complement in $M$. Therefore $X=X_{1} \oplus$ $X_{2} \leq_{e} K_{1} \oplus K_{2} \leq_{c} M$ such that $K_{1} \oplus K_{2} \unlhd_{p} M$. Thereby $M$ is a $P C$-module.

Corollary 1. Let $M=M_{1} \oplus M_{2}$ be a semisimple (or uniform, or injective) module for some $M_{1}, M_{2} \leq M$. If $M_{1}$ and $M_{2}$ are PC-modules, then $M$ is a PC-module.

Proof. It is a consequence of Proposition 3.

Recall that a module $M_{R}$ has the summand sum property, $S S P$, if for all $D_{1}, D_{2} \leq_{d}$ $M_{R}, D_{1}+D_{2} \leq_{d} M$. Motivating SSP definition on complement submodules, $M$ has complement sum property, CSP [7], if for all $K_{1}, K_{2} \leq_{c} M_{R}, K_{1}+K_{2} \leq_{c} M$. Even though the authors in [7] proved that CSP condition implies SSP, we give the following example which shows that the reverse implication of the former property is not true, in general.

Example 3. Let $M$ be the $\mathbb{Z}$-module $\mathbb{Z} \oplus(\mathbb{Z} / \mathbb{Z} p)$ as in Example 1(ii). Note that $\operatorname{Hom}_{\mathbb{Z}}(\mathbb{Z}, \mathbb{Z} / \mathbb{Z} p)=\mathbb{Z} / \mathbb{Z} p$ and $\operatorname{Hom}_{\mathbb{Z}}(\mathbb{Z} / \mathbb{Z} p, \mathbb{Z})=0$.

Thus $M$ has SSP by [15, Exercise 2.41]. On the other hand, we claim $M_{\mathbb{Z}}$ does not have CSP. Assume the contrary. Since $\mathbb{Z}$ and $\mathbb{Z} / \mathbb{Z} p$ are $P C$-modules, $M_{\mathbb{Z}}$ is a $P C$ module by Theorem 3, a contradiction (see, Example 1(ii)). Therefore $M$ does not satisfy CSP.

Now we compose some useful properties of modules with CSP which might help us to consider being Morita invariant property as well as the application to the full matrix rings for the former condition. Let us begin with an easy fact and an example for extending case.

Lemma 3. SSP and CSP conditions are coincide for an extending module.

Proof. It is routine to check.

Example 4. Let $K$ be a field and $R_{R}=\left[\begin{array}{cc}K & K \\ 0 & K\end{array}\right]$. Then $R_{R}$ is an extending module which does not satisfy $C_{3}$. Hence $R_{R}$ does not have SSP. It follows from Lemma 3 that $R_{R}$ does not have CSP. 
Theorem 1. Let $R$ be a ring such that $R=R e R$ and $S=e$ Re for some $e^{2}=e \in$ $R$. Then $M_{R}$ has CSP if and only if the right $S$-module module Me has CSP.

Proof. It is clear from [15, Lemma 2.76 and Proposition 2.77 (ii)].

Corollary 2. Let $R$ be a ring such that $R=R e R$ for some $e^{2}=e \in R$. Then $R_{R}$ has CSP if and only if the right eRe-module Re satisfies CSP.

Proof. It is clear from Theorem 1.

Theorem 2. $M_{n}(R)$ has CSP condition if and only if the free right $R$-module $R^{n}$ has CSP condition.

Proof. Note that $M_{n}(R)=M_{n}(R) e M_{n}(R)$ where $e$ is the matrix unit with 1 in the $(1,1)$-th position and zero elsewhere. Now apply the Theorem 1 and Corollary 2 to get the theorem.

As an application of Theorem 2, it can be easily seen that $M_{2}(\mathbb{Z})$ does not have CSP condition. Indeed, it is renowned that $M_{\mathbb{Z}}=(\mathbb{Z} \oplus \mathbb{Z})_{\mathbb{Z}}$ is an extending module which does not satisfy SSP by [15, Example 2.82]. Hence $M_{\mathbb{Z}}$ does not have CSP by Lemma 3. Thus $M_{2}(\mathbb{Z})$ does not have CSP by Theorem 2. This example explains that CSP condition is not Morita invariant. Now we proceed our main aim of this section.

Theorem 3. Let $M$ be a right $R$-module with CSP such that $M=M_{1} \oplus M_{2}$ for some $M_{1}, M_{2} \leq M$. If $M_{1}$ and $M_{2}$ are $P C$-modules, then $M$ is a PC-module.

Proof. Let $X \unlhd_{p} M$. Then $X=\left(X \cap M_{1}\right) \oplus\left(X \cap M_{2}\right)$ where $X_{1}=X \cap M_{1} \unlhd_{p}$ $M_{1}$ and $X_{2}=X \cap M_{2} \unlhd_{p} M_{2}$ by [5, page 50]. Hence there exist projection invariant submodules $K_{1}$ of $M_{1}$ and $K_{2}$ of $M_{2}$ such that $X_{1} \leq_{e} K_{1} \leq_{c} M_{1}$ and $X_{2} \leq_{e} K_{2} \leq_{c}$ $M_{2}$. Thus $X \leq_{e} K_{1} \oplus K_{2} \leq M$ where $K_{1} \oplus K_{2} \unlhd_{p} M$. Note that $K_{1} \oplus K_{2} \leq_{c} M$ by CSP condition. Thus $M$ is a $P C$-module.

Recall from [13], a module $M$ has $\left(P_{n}\right)$ condition if for every submodule $K$ of $M$ such that $T$ is a direct sum $T_{1} \oplus \cdots \oplus T_{n}$ of complements $T_{i}(1 \leq i \leq n)$ in $M$, every homomorphism $\alpha_{1}: T \rightarrow M$ can be lifted to a homomorphism $\alpha_{2}: M \rightarrow M$. In [13], the authors proved that if $M$ satisfies $\left(P_{n}\right)$ then $M$ satisfies $\left(P_{n-1}\right)$ for all $n \geq 2$. Moreover, they engage a characterization of quasi-continuous modules in terms of $\left(P_{n}\right)$ conditions for every positive integer $n$. Realize that $\left(P_{1}\right)$ does not imply $\left(P_{2}\right)$ (see, [13, Example 10]). The following result spells out $\left(P_{1}\right)$ and $\left(P_{2}\right)$ conditions are equivalent for a module with CSP.

Proposition 4. Let $M$ be a right $R$-module with CSP. Then $M$ has $\left(P_{2}\right)$ condition if and only if $M$ has $\left(P_{1}\right)$ condition.

Proof. Let $M$ be a right $R$-module with CSP. If $M$ has $\left(P_{2}\right)$, then $M$ has $\left(P_{1}\right)$ by [13, page 341]. Conversely, let $M$ has $\left(P_{1}\right)$. Consider $K_{1}, K_{2} \leq_{c} M$ with $\alpha$ : 
$K_{1} \oplus K_{2} \rightarrow M$ homomorphism. Since $M$ has CSP, $K_{1} \oplus K_{2} \leq_{c} M$. Hence $\alpha$ : $K_{1} \oplus K_{2} \rightarrow M$ can be lifted to a homomorphism $\theta: M \rightarrow M$ by the condition of $\left(P_{1}\right)$. Therefore $M$ has $\left(P_{2}\right)$ condition.

We strengthen the characterization of quasi-continuous modules, which is presented in [13], for a module with CSP condition.

Theorem 4. The followings are equivalent for a module $M_{R}$ with CSP.

(1) $M$ is quasi-continuous.

(2) $M$ has $\left(P_{n}\right)$ for every positive integer $n$.

(3) $M$ has $\left(P_{n}\right)$ for some integer $n \geq 2$.

(4) $M$ has $\left(P_{2}\right)$.

(5) $M$ has $\left(P_{1}\right)$.

(6) $M$ is extending.

Proof. (1) $\Leftrightarrow(2) \Leftrightarrow$ (3) $\Leftrightarrow$ (4) Clear from [13, Theorem 4].

(4) $\Leftrightarrow$ (5) It follows from Proposition 4.

(5) $\Leftrightarrow(6)$ Let $M$ has $\left(P_{1}\right)$. Then $M$ has $\left(P_{2}\right)$ by Proposition 4 . Hence $M$ is extending from the fact of (4) $\Leftrightarrow(1)$. Conversely, let $K \leq_{c} M$ and $\alpha: K \rightarrow M$ be a homomorphism. Since $M$ is extending, $K$ is a direct summand of $M$. Consider $g=\pi \iota$ where $\pi: M \rightarrow K$ is projection and $\iota: K \rightarrow M$ is inclusion. It is easy to check that $\left.g\right|_{K}=\alpha$, hence $M$ has $\left(P_{1}\right)$.

\section{REFERENCES}

[1] F. W. Anderson and K. R. Fuller, Rings and Categories of Modules. New York: Springer, 1974.

[2] G. F. Birkenmeier, J. K. Park, and S. T. Rizvi, "Modules with fully invariant submodules essential in fully invariant summands,", Comm Algebra, vol. 30, pp. 1833-1852, 2002, doi: 10.1081/AGB120013220.

[3] G. F. Birkenmeier, A. Tercan, and C. C. Yücel, "The extending condition relative to sets of submodules," Comm Algebra, vol. 42, pp. 764-778, 2014, doi: 10.1080/00927872.2012.723084.

[4] N. V. Dung, D. V. Huynh, P. F. Smith, and R. Wisbauer, Extending Modules. London: Pitman, 1994.

[5] L. Fuchs, Infinite Abelian Groups I. New York: Academic Press, 1970.

[6] K. R. Goodearl, Ring Theory: Nonsingular Rings and Modules. New York: Dekker, 1976.

[7] I. M. Hadi and T. Y. Ghawi, " Modules with the closed sum property," Int. Math. Forum, vol. 9, no. 32, pp. 1539-1551, 2014, doi: 10.12988/imf.2014.48151.

[8] Y. Kara and A. Tercan, " Modules whose certain submodules are essentially embedded in direct summands," Rocky Mt. J. Math., vol. 46, no. 2, pp. 519-532, 2016, doi: 10.1216/RMJ-2016-462-519.

[9] Y. Kara and A. Tercan, "On the inheritance of the strongly $\pi$-extending property," Comm Algebra, vol. 45, no. 8, pp. 3627-3635, 2017, doi: 10.1080/00927872.2016.1243245.

[10] T. Y. Lam, Lectures on Modules and Rings. Berlin: Springer, 1999.

[11] S. Mohammed and B. J. Müller, Continuous and Discrete Modules. Cambridge: Cambridge Univ. Press, 1990.

[12] P. F. Smith, "Modules for which every submodule has unique closure," Proc. Biennial OhioDeniso Conf., pp. 302-313, 1993. 
[13] P. F. Smith and A. Tercan, "Continuous and quasi-continuous modules," Houston J. Math., vol. 18, no. 3, pp. 339-348, 1992.

[14] P. F. Smith and A. Tercan, "Direct summands of modules which satisfy $C_{11}$," Algebra Colloq., vol. 11, pp. 231-237, 2004.

[15] A. Tercan and C. C. Yücel, Module Theory, Extending Modules and Generalizations. Basel: Birkhäuser, 2016.

\section{Author's address}

\section{Yeliz Kara}

Uludag University, Department of Mathematics, Görükle, 16059 Bursa, Turkey

E-mail address: yelizkara@uludag.edu.tr 